\title{
Localization of Folds and Cracks in Thin Metal Films Coated on Flexible Elastomer Foams
}

\author{
Hugues Vandeparre, Qihan Liu, Ivan R. Minev, Zhigang Suo, and Stéphanie P. Lacour*
}

Folds and cracks are familiar motifs in biological and synthetic coatings. They arise from a mechanical mismatch between a stiff sheet bonded to a compliant substrate. The sheet forms wrinkles under compressive stress ${ }^{[1-4]}$ and forms cracks under tensile stress. ${ }^{[5-7]}$ Self-organized patterns of folds and cracks have been exploited in a number of applications, including nano/microfabrication, ${ }^{[8]}$ surface and interface science, ${ }^{[9,10]}$ biotechnology, ${ }^{[11]}$ photonics $^{[12]}$ and stretchable electronics. ${ }^{[13,14]}$

Here, we report on a thin metal film coated on a microcellular elastomer foam. When the substrate is stretched, folds and cracks form in the metal film, but they are confined in localized regions. Outside these regions, the metal film remains crack-free and fold-free, leading to percolating paths for electrical conduction. The hybrid metal film on elastomer foam is a highly conformal conductor, capable of large stretch, dramatic changes in shape without impairing its electrical functionality.

Figure 1 illustrates the localization of cracks and folds in a stretched metal film on a microcellular elastomer substrate. The cellular substrates used in this study areflexible polyurethane foams (FPFs). They consist of gas-filled cells (e.g., air or $\mathrm{CO}_{2}$ ) distributed in polyurethane (PU). The cellular microstructure arises spontaneously during the formation of the PU foam due to the effect of the blowing agent. The surface of the foam is smooth, and the cells are spontaneously covered with a thin layer of PU. On the surface of the foam, we evaporate a thin, sub-micrometer thick gold film. The hybrid structures are stretched uniaxially to applied strains $\left(\varepsilon_{\text {appl }}\right)$ of 20 and $50 \%$. For comparison, we also evaporate the gold film on a plain PU elastomer. Prior to mechanical loading, the gold films on both the foam and plain elastomers are uniform and smooth across its entire surface area.

When the metal film on a plain elastomer is stretched, the film forms a uniform distribution of cracks perpendicular to the stretching direction, and wrinkles normal to the cracks (Figure 1a). The wrinkles parallel to the tensile axis arise from Poisson compression. When the metal film on an elastomer foam is stretched, however, a non-uniform pattern of

Dr. H. Vandeparre, Dr. I. R. Minev, Prof. S. P. Lacour Ecole Polytechnique Fédérale de Lausanne (EPFL) Centre for Neuroprosthetics

STI | IMT/IBI | LSBI, CH-1015 Lausanne, Switzerland E-mail: stephanie.lacour@epfl.ch

Q. Liu, Prof. Z. Suo

School of Engineering and Applied Sciences

and Kavli Insitute for Bionano Science and Technology

Harvard University

Cambridge, MA 02138, USA

DOI: 10.1002/adma.201300587 cracks and folds develops. Cracks and folds form in the gold film above the large cells, leaving other regions of the gold film crack-free and wrinkle-free. The spacing between the cracks, $\lambda_{\text {crack }}$, is smaller than the spacing of the cracks in the gold film on the plain PU substrate, while the width of the individual cracks, $w_{\text {crack}}$, is smaller. Figure $1 c$ displays the local strain in the gold film on the plain PU and the local strain in the gold film above one cell on the foam. The local strain is extrapolated from the crack distribution in scanning electronic microscopy SEM images. At $\varepsilon_{\text {appl }}=20 \%$, the local strain in the metal film on the plain PU is uniform and equal to $\varepsilon_{\text {appl }}$. On the PU foam, the strain above the cell peaks to nearly twice the applied strain, and remains close to $0 \%$ in between the cells. Furthermore, instead of releasing the Poisson compression by forming periodic wrinkles, the film develops large folds, constrained above the cells (Figure 1b). Larger applied strain forces these wrinkles merge into a single fold (Figure 1d), while the surrounding gold film is intact. ${ }^{[15-17]}$

Sub-millimeter thick membranes of PU foam are produced using a simple bar coater setup (Figure 2a). A variety of two-component systems are available to produce PU foams, allowing for a wide range of foam densities and morphologies. To study the influence of the substrate cellular structure, foams are prepared with two distinct densities, 0.1 and $0.4 \mathrm{~g} / \mathrm{cm}^{3}$, corresponding to tensile moduli of $80 \mathrm{kPa}$ and $500 \mathrm{kPa}$, respectively (Figure $2 \mathrm{~b})$. The plain PU membrane $\left(\rho=1.2 \mathrm{~g} / \mathrm{cm}^{3}\right)$ has a modulus of $6 \mathrm{MPa}$. Figure 2c,d display 3D micro computed tomography $(\mu \mathrm{CT})$ reconstructions of the stiff and soft foams, with a vertical growth direction. The microcellular substrates are open-celled foams sandwiched between very thin PU surface skins $(<100 \mu \mathrm{m}$ thick) (Figure 2e). The cells occupy most of the foam volume. Despite the surface skin, the cells are clearly visible from the top surface of the elastomeric foam (Figure 2f). Due to the preparation conditions, the foams present structural anisotropy, and the cells are elongated in the vertical direction (Figure 2e). The average cell-wall width $t$, height $H$ and cell width $W$ are summarized for both foam densities (Figure 1g).

Strain maps of the foam surface are obtained using a video extensometer. Peak strain zones above the cells are clearly visible Figure 3a. We model the cells and their packing with a hexagonal arrangement, and define four regions at the surface of the foam: zone A highlights V-shaped stripes above the cell walls oriented at an angle of approximately $60^{\circ}$ to the $\varepsilon_{\text {appl }}$ direction; zone $\mathrm{B}$ corresponds to stripes above the cell walls parallel to $\varepsilon_{\text {appl }}$ direction; zone $\mathrm{C}$ is the area above the cell, delimited by and normal to zones $\mathrm{B}$; and zone $\mathrm{D}$ is the remaining area. The strain in each zone (averaged from the stain maps) is plotted Figure $3 \mathrm{~b}, \mathrm{c}$ as a function of $\varepsilon_{\text {appl }}$. The strain along the cell walls (zones A \& B) is significantly lower than that above the cell (zones $C \& D$ ) and than $\varepsilon_{\text {appl }}$. The data show a more 
a

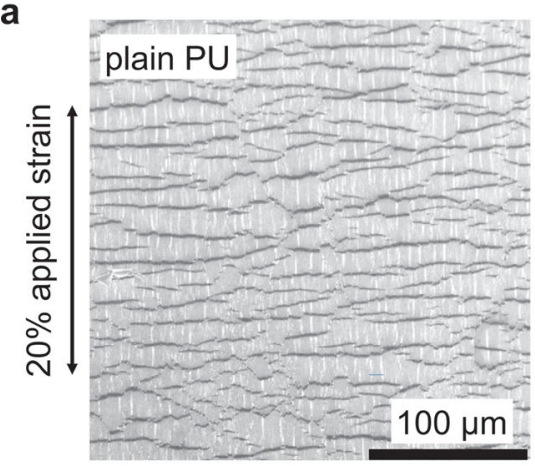

C

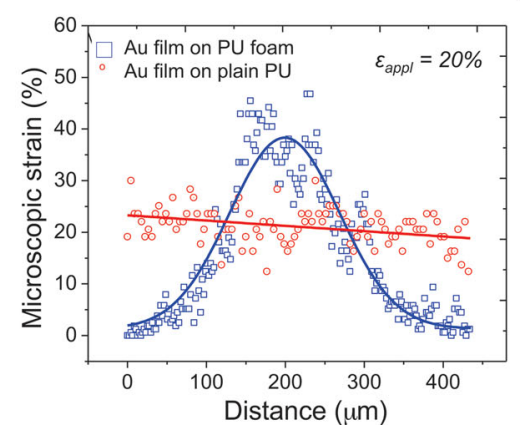

b

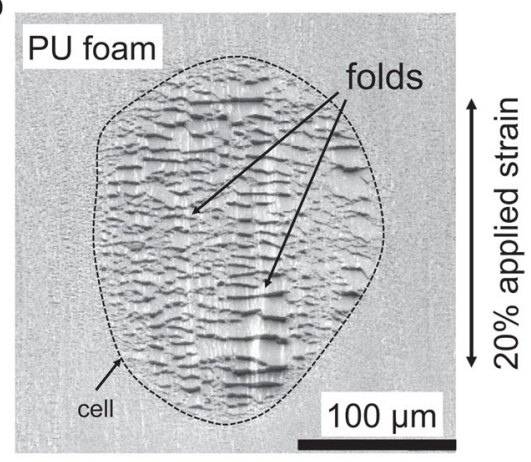

d

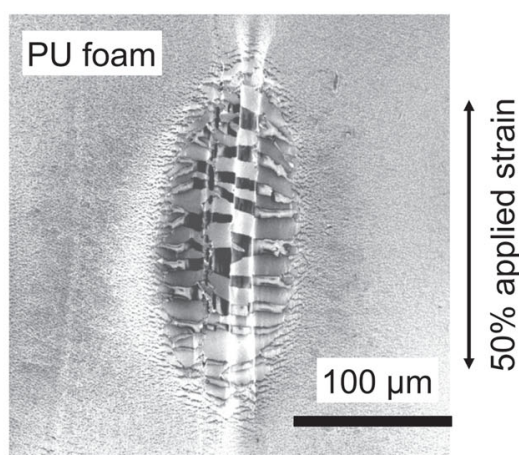

Figure 1. Thin gold film on a polyurethane elastomer and on polyurethane foams. Scanning electron micrographs (SEM) of a $25 \mathrm{~nm}$-thick gold film evaporated on: a) a plain PU elastomer $\left(\rho=1.2 \mathrm{~g} \mathrm{~cm}^{-3}\right)$ and $\left.\mathrm{b}\right)$ on a microcellular PU foam $\left(\rho=0.1 \mathrm{~g} \mathrm{~cm}^{-3}\right)$, held at an applied strain of $20 \%$. c) Local strain in the gold film above a cellular PU foam substrate and above plain PU elastomer when the structure is stretched by $20 \%$ strain. The strain is derived from the local density of cracks and plotted in the direction normal to the applied strain. d) SEM of a $25 \mathrm{~nm}$ thick gold film on a PU foam $\left(\rho=0.1 \mathrm{~g} \mathrm{~cm}^{-3}\right)$ held at an applied strain of $50 \%$.

www.MaterialsViews.com

pronounced strain anisotropy in the soft foam than in the stiff foam. Due to experimental resolution, it is not possible to observe the splitting of the strain in zones $\mathrm{C}$ and $\mathrm{D}$ in the case of the stiff foam (Figure 3c).

To quantify the strain levels at the surface of the foam further, we simulate the deformation of the structure as in Figure 3d under elongation using the finite element method. The foam is modeled as an array of hexagonal cells with cell-wall length $l$ and width $t$. The angle between the two cell walls is $\Theta=120^{\circ}$ before the structure is stretched. The thin elastomeric skin above the cells is neglected in the simulation. The maximum elongation of the cell can be readily estimated by using a rigid-body rotation model, in which the cell walls rotate towards the tensile axis without stretching. This idealized model yields a maximum relative unit cell deformation of $1 / 3$. In elastomeric honeycombs, the rotation of the cell walls leads to bending in the cell walls of zones A (Figure 3e). ${ }^{[18]}$ This mode of deformation is favored for small relative cell-wall thicknesses $t / l$, since the bending and stretching stiffness scales as $\left(\frac{t}{t}\right)^{3}$ and $\frac{t}{I}$, respectively. As a result, the smallest strains are observed in zones A (Figure 3b), and larger strains appear in zone B cell walls, which are not able to bend. When the thickness of the cell wall is larger a

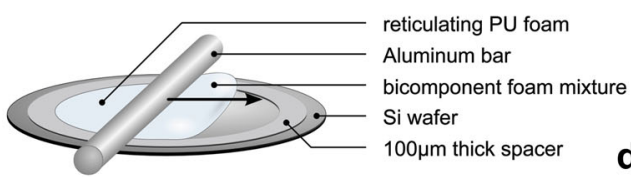

b

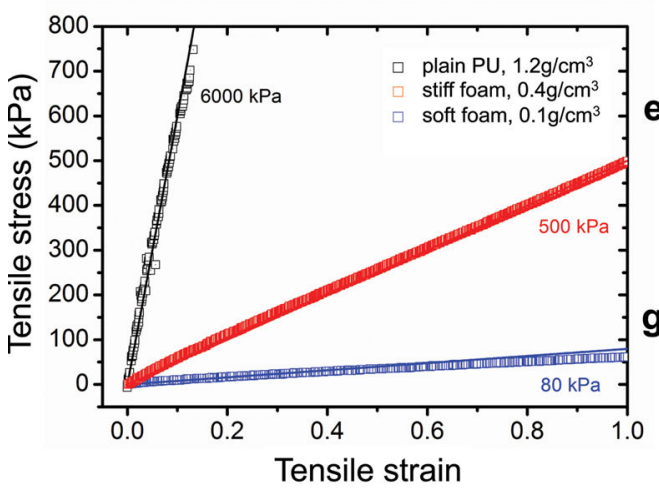

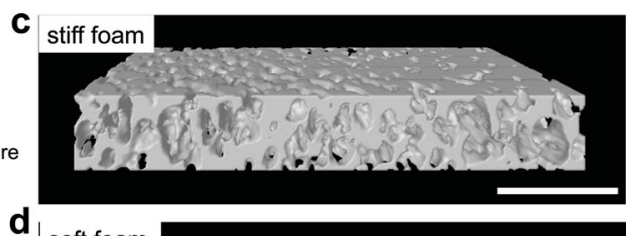

d

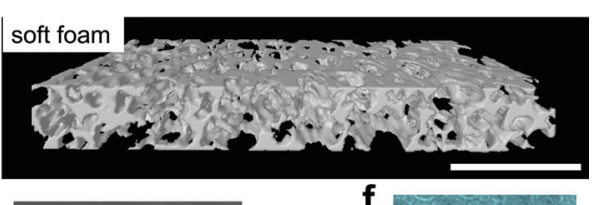

e

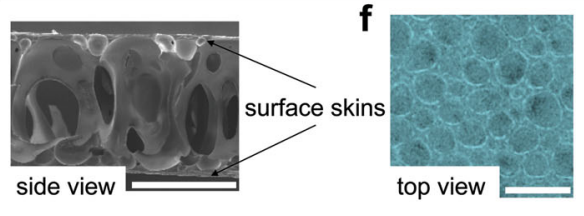

g

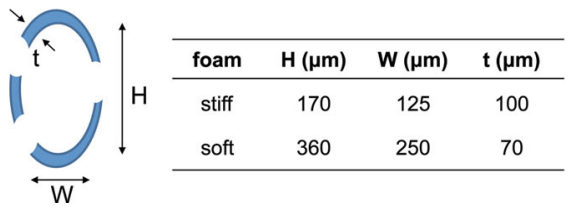

Figure 2. Morphology and elastic modulus of PU foams. a) Schematic of the fabrication of a sub-millimeter thick PU foam membrane. b) Tensile stress-strain curves and corresponding elastic modulus of plain PU elastomer, stiff and soft PU foams. c,d) Micro Computed Tomography ( $\mu$ CT) scans of stiff $\left(\rho=0.4 \mathrm{~g} \mathrm{~cm}^{-3}\right)$ and soft $\left(\rho=0.1 \mathrm{~g} \mathrm{~cm}^{-3}\right)$ PU foams; scale bar: $1 \mathrm{~mm}$. e) Side view of a soft $\left(\rho=0.1 \mathrm{~g} \mathrm{~cm}^{-3}\right)$ PU foam obtained by scanning electron microscopy; scale bar: $0.5 \mathrm{~mm}$. f) Top view of a soft $\left(\rho=0.1 \mathrm{~g} \mathrm{~cm}^{-3}\right)$ PU foam; scale bar: $0.5 \mathrm{~mm}$. g) Schematic of an open cell with the vertical axis aligned with the foam rise direction labeled with the cell's height $H$, width $W$ and wall thickness $t$; the table reports the corresponding dimensions measured from the $\mu \mathrm{CT}$ scans for both stiff and soft PU foams. 

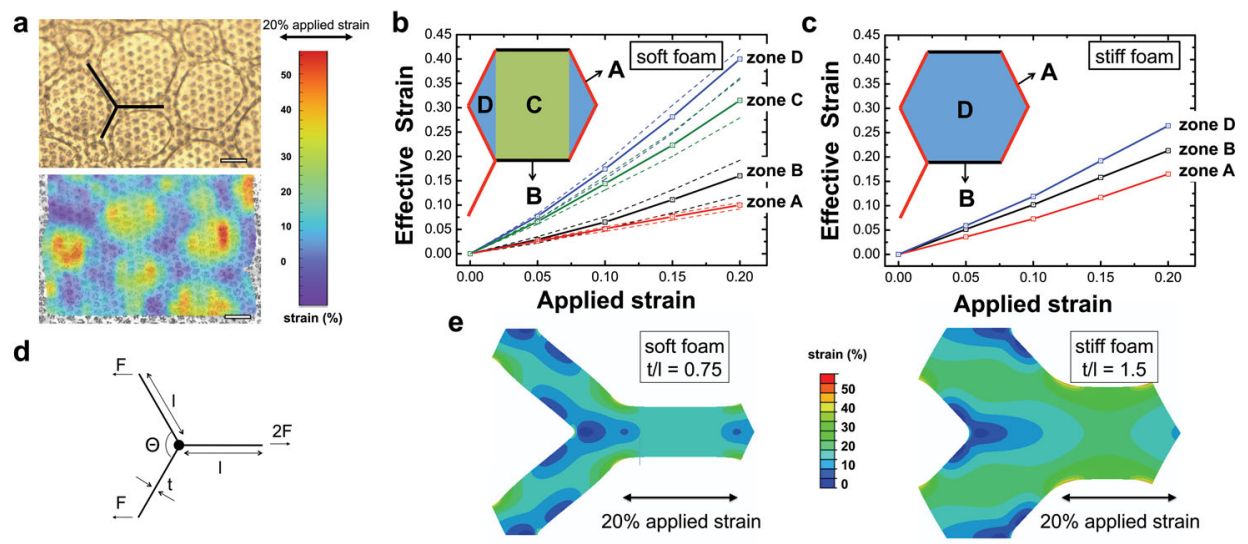

Figure 3. Surface strain mapping. a) Optical image and corresponding strain map measured at the surface of a soft PU foam stretched along the $x$-axis to an applied strain of $20 \%$; scale bar: $100 \mu \mathrm{m}$. b,c) Average strains experimentally determined in the four zones A to D at the surface of the soft (b) and stiff (c) foam as a function of the applied strain; the schematic illustrates each zone above one cell. d) Parameter model of the cell walls arranged in ideal hexagonal packing. e) Finite element simulation of the surface strain across the cell walls for a soft and stiff foam stretched macroscopically to an applied strain of $20 \%$.

than its length, $t / l>1$, the contribution of the stretching mode dominates and the rotation of the cell walls is hindered.

Furthermore, we observe that the strain in the freestanding PU skin covering the honeycomb network (in zones C \& D) is much larger than $\varepsilon_{\text {appl }}$ (Figure $3 \mathrm{~b}, \mathrm{c}$ ). This results from a stretching mode rather than bending mode as the thin, elastic skin is forced to accommodate the surrounding cell walls rotation and stretch.

Affine deformation in an elastomer is usually considered at the level of the local chains arrangement. Here we not only have an elastomer but also pores. The local strain is strongly affected by the heterogeneity at the scale of pore size. This large departure from affine deformation is analogous to that in granular materials. ${ }^{[19-20]}$

The microcellular elastic substrates are used to produce highly stretchable thin metal film conductors. Figure 4a summarizes the electromechanical response of gold film stripes patterned on plain PU, stiff and soft PU foam substrates. All foam samples exhibit large stretchability and conformability, and can sustain repeated mechanical loading. The electrical resistance of the stretchable conductors as a function of the number of stretch cycles is presented Figure $4 \mathrm{~b}$. The sample's electrical resistance is recorded at $0 \%$ applied strain immediately after a cycle, and at $20 \%$ applied strain. Resistance values are very stable over the 1000 cycles. All foam samples exhibit large stretchability and conformability (Figure 4c) a

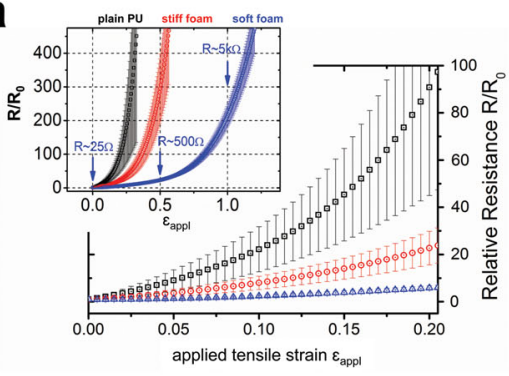

b
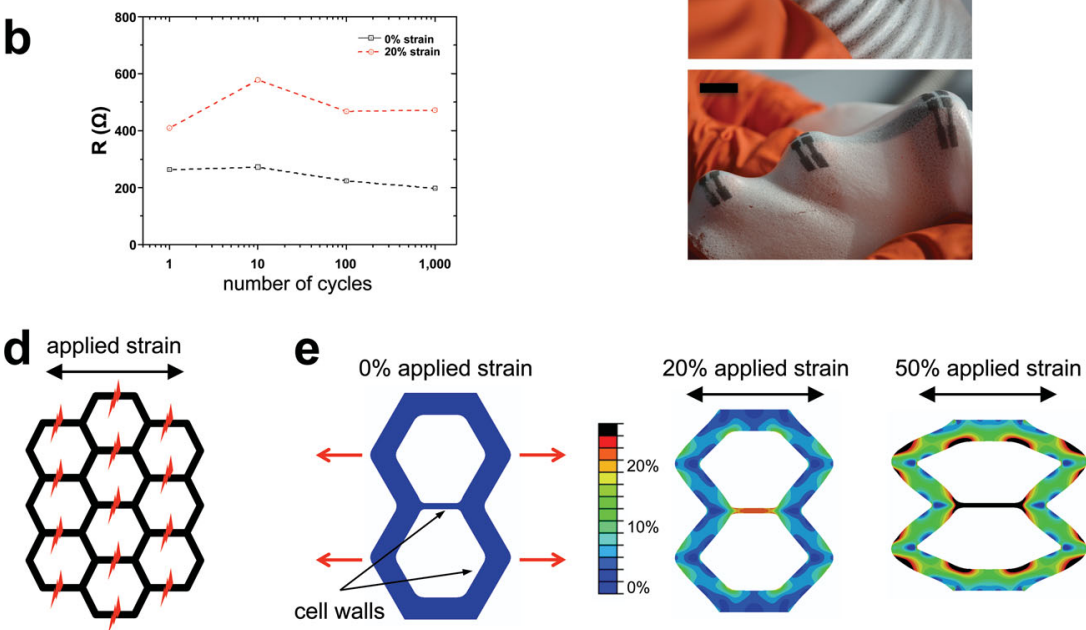

Figure 4. Stretchable thin gold film on flexible PU foam. a) Relative electrical resistance as a function of applied strain of a $25 \mathrm{~nm}$-thick, $5 \mathrm{~mm}$-wide, $20 \mathrm{~mm}$-long gold film evaporated on $0.5 \mathrm{~mm}$ thick plain PU (black), stiff PU foam (red) and soft PU foam (blue); inset: all samples are stretched to large strains before failure. b) Electrical resistance (recorded at $0 \%$ and $20 \%$ strain) as a function of number of stretching cycles to $20 \%$ strain. Conductors are $25 \mathrm{~nm}$ thick, $5 \mathrm{~mm}$ wide, $20 \mathrm{~mm}$ long. c) Optical images of highly conformal gold conductors on PU foam; scale bar: $5 \mathrm{~mm}$. d) Schematic of an ideal hexagonal packing of the cells in a foam illustrating mechanical failure along the thin walls parallel to the applied strain direction thereby preventing any electrical percolating path in the gold film. e) Finite element simulation of the top surface strain across two adjacent hexagonal cells at $0 \%, 20 \%$ and $50 \%$ applied strain. 
and can sustain repeated mechanical loading (data not shown). The conductors' maximum elongation $\varepsilon_{\max }$ (defined when the relative change in resistance is larger than 500) improves with the relative softness of the substrate: $\varepsilon_{\text {max, plain }}=25 \%<\varepsilon_{\text {max,stiff }}=$ $55 \%<\varepsilon_{\text {max, soft }}=120 \%$. Furthermore the relative change in resistance is minimal when the metal film is deposited on the softest foam. For a given $\varepsilon_{\text {appl }}$, the large strain fields above the cells induce catastrophic cracking and folds in the gold film but at the same time prevent or delay any cracking in the surrounding gold ligaments, which allows for a stable percolating path thus continuous electrical conduction.

The large stretchability observed in the experiment is remarkable. When the foam is modeled as an array of identical cells (Figure 4d), all cell walls aligned with the stretching direction (i.e., in zone B) will rupture simultaneously. Note that the failure strain of the gold film on plain PU is about $\varepsilon_{\text {max, plain }} \approx 25 \%$ (Figure $4 \mathrm{a}$ ), and the effective strain in zones $\mathrm{B}$ in the gold film on the soft foam is $\varepsilon_{\text {eff }}^{\text {zone } B} \approx \frac{3}{4} \varepsilon_{\text {appl }}$ (Figure $3 b$ ). Consequently, the idealized model would predict that the failure strain of the gold film on the soft foam, $\varepsilon_{\text {max,soft }}$, is at most $+33 \%$. However, experimentally measured value is significantly larger, $\varepsilon_{\text {max,soft }} \approx 120 \%$ (Figure $4 \mathrm{a}$ ). This difference can be explained by the actual morphology of the foam microstructure: the cell arrangement is not ideally hexagonal, but rather random. As a result, the strains in the cell walls have a wider distribution than in the idealized honeycombs. ${ }^{[18]}$ In Figure 4e, we conduct a 2D FEM simulation for two neighboring hexagonal cells with a central cell wall $(t / l=0.2)$ thinner than the surrounding ones $(t / l=0.75)$, and stretched to 20 and $50 \%$ $\varepsilon_{\text {appl. }}$. The narrower walls suffer from much larger strain than their wider counterparts. However, the excessive stretch of the narrow wall makes the rotation within one unit cell asymmetric thus helps relieving more effectively $\varepsilon_{\text {appl }}$ across the foam structure thus maintaining a continuous percolating path, even at $\varepsilon_{\text {appl }} \gg \varepsilon_{\text {max, plain }}$.

Thin gold stripes on top of heterogeneous elastomer substrate retain their conductance during stretching and relaxation. The orientation of the gold stripes with regard to the stretching direction does not affect their overall stretchability. This is mainly due to the random nature of the foam microstructure. Oriented or aligned microstructures (cells) in the polyurethane matrix would favor specific stretching directions thus limit the interconnect design (Figure 4d). However, one limiting factor should be accounted for: the minimum feature size of the conductor (i.e., its width) should also be significantly larger than the average pore (cell) size.

Folds and cracks are mechanical instabilities in thin films that occur over large areas when soft matter with hard skins are stretched or compressed. On microcellular elastomeric substrates, folds and cracks localize above the cells leave a nearly undamaged film between them. Of particular interest is the use of this localized damage to expand the electromechanical performance of conductors. This approach offers a straightforward, non-lithographic route to extend the stretchability of thin metal films. So far, we have focused on gold conductors on polyurethane foams, but other material combinations might open the way for the integration of intrinsically brittle materials in flexible and stretchable electronics.

\section{Experimental Section}

Substrate Preparation: Two-component mixture, flexible polyurethane foams were purchased from Smooth-On. FlexFoam-it III and FlexFoam-it 17 were used to form the soft and stiff foam substrates, respectively. In both cases, the two-component mixture was dispensed on a glass wafer, and spread with a bar coater between spacers of a pre-defined thickness. A smooth polyimide foil covered the foam during spreading; an overpressure can be applied above the foam film to constrain its vertical expansion. This ensured a fine control of the thickness and density of the foam. Both the polyimide foil and glass wafer were coated with Ease Release 2831 (Smooth-on) to ensure easy peeling-off of the cross-linked foam membranes.

Thin Metal Film Deposition: Bi-layers of titanium (Ti, $5 \mathrm{~nm}$ ) and gold $(\mathrm{Au}, 25 \mathrm{~nm}$ ) films were deposited on the flexible foam substrate. The Ti layer was electron-beam evaporated then the gold film was thermally evaporated without breaking the vacuum. The adhesion of the metal films on the soft foam successfully passed tape peeling tests. The metallic conductors were patterned through a polyimide shadow mask positioned directly on the surface of the foam substrate during evaporation.

Surface Strain Mapping: A video extensometer was used to monitor the strain at the surface of the samples. The latter were mounted in a customized uniaxial stretcher where strains $\varepsilon_{\text {appl }}$ of $0,5,10,15$, and $20 \%$ were applied. Optical images of the sample surface were recorded using a Keyence digital microscope $\mathrm{VHX600} \mathrm{with} \mathrm{ultra} \mathrm{long} \mathrm{working} \mathrm{distance}$ objective. Printer toner powder was dispersed on the foam surface through a TEM grid used as a mask. The grid was then removed leaving a regular pattern. The distortion of the pattern upon mechanical loading was used to compute the strain fields at the surface of the samples. The position of particles in the pattern was tracked using the Istra 4D v4.3.0 software. The change in the distance between the particles was monitored as the sample is stretched from $0 \%$ to $20 \%$ and the resulting microscopic strain was calculated. The strain was calculated at 238 points distributed in a square grid (the grid spacing is approximately $15 \mu \mathrm{m})$ across the image. The color map was interpolated between the points.

Scanning Electron Microscopy and Local Strain Measurement: Samples were stretched to 0,20 , and $50 \%$ strain and glued (stretched) to a glass slide. No additional coating was added to the metallized foams prior to imaging in the Zeiss Merlin scanning electron microscope. Using Image J, the images were converted to binary format. Each pixel was labeled either as being inside a crack or being "intact" gold film. Once the metal film on PDMS is cracked, we assume sample elongation is accommodated by crack widening (as the elastomer is much softer than the gold film).

Electro-mechanical Characterization: The conductors were mechanically cycled using a home-built, computer-controlled, and motorized uniaxial stretcher. The long axis of the conductor was aligned with the stretching direction so that the elongation of the gold thin film is maximal in the applied strain direction. The electrical resistance of the gold film was monitored with two point contacts positioned at both ends of the gold stripes using a Keithley 2400 sourcemeter. During prolonged cycling to $20 \%$ applied strain, the flexible polyurethane foam showed expected creep; the samples were thus repositioned flat after hundreds of cycles.

Finite Element Modeling: 2D finite element simulations were conducted with the commercial code ABAQUS under the plane stress conditions. The elastomer was modeled as a neo-Hookean material. In Figure 3, periodic boundary condition was applied and the applied strain was $20 \%$. All corners were rounded with a radius of curvature equal to half of the thickness of the cell wall. The color scale used in simulation matched that of the experimental mapping (Figure 3a). In Figure 4, the outer cell wall had a $\mathrm{t} / \mathrm{l}$ ratio of 0.75 while the inner cell wall was modeled with a $t / l$ ratio of 0.2 . Corners were rounded similarly to Figure 3 . The horizontal displacement was forced to be uniform on the left and right boundaries. A uniform traction is applied at the intersections of two hexagonal cells as shown Figure 4e. 


\section{Acknowledgements}

This work was supported by the Bertarelli Foundation, and a European Research Council Starting Grant, ERC, no. 259419. H.V. and S.P.L. thank Prof. V. Michaud, Prof. D. Pioletti and their collaborators for fruitful discussions. Q.L. and Z.S. acknowledge the support of the National Science Foundation Materials Research Science and Engineering Center.

Received: February 4, 2013

Revised: March 12, 2013

Published online:

[1] N. Bowden, S. Britain, A. G. Evans, J. W. Hutchinson, G. M. Whitesides, Nature 1998, 393, 146.

[2] E. Cerda, L. Mahadevan, Phys. Rev. Lett. 2003, 90, 074302.

[3] S. Périchon Lacour, S. Wagner, Z. Huang, Z. Suo, Appl. Phys. Lett. 2003, 82, 2404.

[4] H. Vandeparre, J. Léopoldès, C. Poulard, S. Desprez, G. Derue, C. Gay, P. Damman, Phys. Rev. Lett. 2007, 99, 188302.

[5] T. Li, Z. Huang, Z. Suo, S. P. Lacour, S. Wagner, Appl. Phys. Lett. 2004, 85, 3435

[6] N. E. Janssens, Y. Leterrier, J.-A. E. Manson, Eng. Fract. Mech. 2006, 73, 2614.
[7] N. J. Douville, Z. Li, S. Takayama, M. D. Thouless, Soft Matter 2011, 7, 6493.

[8] K. H. Nam, I. H. Park, S. H. Ko, Nature 2012, 485, 221.

[9] E. P. Chan, E. J. Smith, R. C. Hayward, A. J. Crosby, Adv. Mater. 2008, 20, 711.

[10] S. Yang, K. Khare, P.-C. Lin, Adv. Funct. Mater. 2010, 20, 2550.

[11] X. Zhu, K. L. Mills, P. R. Peters, J. H. Bahng, E. H. Liu, J. Shim, K. Naruse, M. E. Csete, M. D. Thouless, S. Takayama, Nat. Mater. 2005, 4, 403

[12] J. B. Kim, P. Kim, N. C. Pégard, S. J. Oh, C. R. Kagan, J. W. Fleischer, H. A. Stone, Y-L. Loo, Nat. Photonics 2012, 6, 327.

[13] J. A. Rogers, T. Someya, Y. G. Huang, Science 2010, 327, 1603.

[14] E. Delivopoulos, D. Chew, I. R. Minev, J. W. Fawcett, S. P. Lacour, Lab Chip 2012, 12, 2540.

[15] L. Pocivavsek, R. Dellsy, A. Kern, S. Johnson, B. Lin, K. Y. C. Lee, E. Cerda, Science 2008, 320, 912

[16] F. Brau, H. Vandeparre, A. Sabbah, C. Poulard, A. Boudaoud, P. Damman, Nat. Phys. 2010, 7, 56.

[17] P. Kim, M. Abkarian, H. A. Stone, Nat. Mater. 2011, 10, 952.

[18] L. J. Gibson, M. F. Ashby, in Cellular Solids Structure and Properties, 2nd edition (Eds: D. R. Clarke, S. Suresh, I. M. Ward, FRS), Cambridge University Press, Cambridge, UK 1997, Ch. 4.

[19] B. Utter, R. P. Behringer, Phys. Rev. Lett. 2008, 100, 208302.

[20] A. Tordesillas, M. Muthuswamy, S. D. C. Walsh, J. Eng. Mech. 2008, 134, 1095. 